\title{
Study on Interaction of Restoration and Experience of Cultural Heritage Based on Event in Virtual Environment
}

\author{
Kiwan $\mathrm{Ko}^{1}$ and Jeongik Min ${ }^{2 *}$ \\ ${ }^{1,2}$ Jeonju University, College of Culture Convergence \\ ${ }^{1}$ fishgo@jj.ac.kr, ${ }^{2}$ minji@jj.ac.kr
}

\begin{abstract}
There is a lot of research on the restoration of artifacts and on techniques for utilizing artifacts, but research on the backgrounds and construction process of cultural heritage and their applications has been limited. For restoration techniques, restoration of cultural heritage through $3 D$ scanning has been the main research subject. In the case of a destroyed cultural heritage where there is no $3 D$ scan data and there is only a simple historical record and remains, it is impossible to reconstruct it digitally. In this situation, other reconstruction methods should be studied. A lot of the cultural heritage of countries is in this type of situation due to wars, earthquakes, disasters, etc., and much of the cultural heritage designated by UNESCO is in this situation. This study is meaningful in restoring lost cultural heritage, and this study can be differentiated from existing research by its use of game techniques in a virtual environment., Precision, precision of graphics, and correspondence with historical records are important factors in restoration using game techniques in a virtual environment. After completing such reconstruction, the scope of this research was extended to experience cultural heritage like reality and to utilize it. This study also includes the character of the fusion of graphic theory, interactive theory, and game theory.
\end{abstract}

Keywords: VR contents, interactive, digital heritage, event modeling, mapping

\section{Introduction}

Digital restoration, digital display, and the experience of cultural heritage are progressing through the research and development of various digital technologies such as 3D scanning, 3D printing, virtual reality, and augmented reality. Although many studies have been actively researched to restore and preserve cultural heritage, research on the use of cultural heritage is insufficient. The possibilities of digital cultural heritage should be sought to preserve the information about the prototype of the cultural heritage and to utilize it in a new form [3][10]. UNESCO defines digital heritage as a digital version of cultural heritage information such as historical sites, cultural, aesthetic, archaeological and scientific values, relics and buildings, objects and intangibles, or a digital conversion of the analog form of cultural heritage. It is defined as the preservation of cultural heritage and should be developed to discover new values of digital heritage and to make new experiences possible. In other words, it should be extended to record cultural heritage and to use cultural heritage sharing and promotion in reproduction.

This thesis focuses on the direction and technology of advanced processing methods of restoring lost cultural heritage in a virtual environment by graphical processing techniques, image processing techniques and game techniques in a virtual environment in terms of fusion of cultural heritage restoration and cultural heritage utilization. It can be extended to the ways of reproducing cultural heritage and experiencing technology, and to utilizing acquired data effectively. Research has been conducted with the technology of

* Corresponding Author 
discovering and digitizing cultural heritage [5], prototype modeling restoration technology based on digitally measured data [3,9], digital museum technology for displaying digital cultural heritage, and story-based cultural heritage exploration technology through interactive media. The scope of this study is based on the existing cultural heritage. This paper is meaningful in the study of what does not exist now and what remains unsecured. In this case, restoration based on historical evidence is combined with restoration of scanning technology and modeling technology. It can be said that this restoration method widens the degree of utilization of the restoration according to the part of the reality in the reconstructed virtual environment image $[10,11]$ and the degree of reflection of the reality attribute of the reconstructed space [7, 9]. Based on this, it is possible to define the shape, surface, and color attributes as a formative element by analyzing the visual reality implementation elements of 3D VR contents according to the level of importance while considering the actual level $[4,5]$. Modeling elements are involved in the process of modeling(shape), mapping(texture), lighting(Threedimensional feeling), and animation(visual story) during the 3D animation production process $[1,6]$. In this paper, we compare the present and past of reconstructed heritage by combining 3D scanning, VR, and imaging techniques, and eliminate the problems of screen dizziness during VR movement and research, and program it to enable history experience through events.

There is a case of restoring cultural heritage and expanding into a new industry by integrating contents with smart devices of traditional culture. It is the Michelangelo project that started in 1997 with the United States and Italy. Based on three-dimensional precision measurement and modeling technology, it was used to measure ancient buildings such as the Tower of David, ancient Rome map. In the London Museum, there was an attempt to construct a content that made historical experience possible by displaying present and past images in a 3D image when clicking on a certain place in London through the Street Museum service. In the Louvre Museum, the Louvre-DNP Museum App uses 3D and augmented reality to allow users to experience the monastery before being destroyed by the war. This approach can be used to reconstruct existing cultural heritage objects accurately, but there is a limit to its ability to restore lost cultural heritage. Precision graphics required for the restoration of the cultural heritage lost due to the generation of high-quality data necessary for restoration of cultural heritage and the reconstruction of cultural heritage lost by the combination of molding elements, and experience of traditional culture by making precise contents from the buildings around is a research method for advanced modeling that can be experienced by comparing with the current state.

In order to reconstruct and model experiences, we used satellites to reproduce the past scenes of cultural sites. Satellite images were taken to capture the location of the cultural relics in the actual location, the terrain of the surrounding environment, and so on. We set the scope of work to realize the current image of the cultural ruins by shooting the current figure using a drone at various angles. We present a technique to embody a game engine in modeling that interacts with contents in real time without gazing at hardware other than HMD.

\section{Approach of Modeling}

In this paper, we propose a method of real work area planning using satellite image map and Heightmap extraction, a method of determining working range through actual location size (drone shooting), generation of actual restoration data, modeling for application of real time realization method and development method of VR contents using the Game Unity engine. It is a methodology that can recover and utilize the cultural heritage that is lost by such a development method, that is, in cases where 3D scanning data is impossible. 


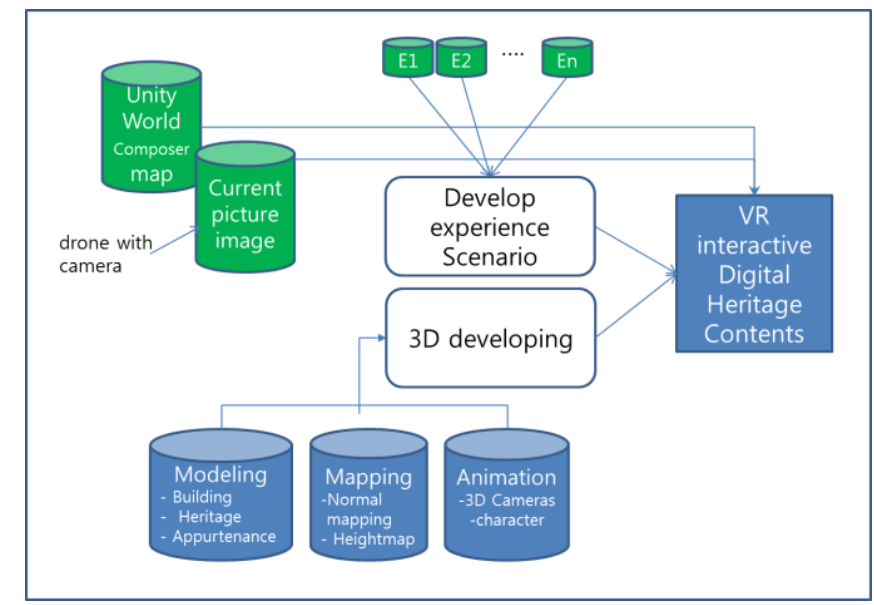

Figure 1. Framework of Real-time Interactive VR

The VR developed through this methodology can be utilized through various experiential scenarios. Various events needed to experience the historical background and experience interactive VR contents using thinking concepts and cognitive abstraction from simple visual information are provided.

Table 1. Digital Heritage Experience Scenario Sample

-Realizes the 360 degree video and real-time VR 3D
space, which is the only one among the existing
heritage-related VR contents, in the same contents
seamlessly.
- Wide range of cultural heritage photographs and
2km surroundings are implemented. In the example
Work, the satellite photographs are edited and
reconstructed.
- Fine-grained modeling and map source work that
reproduces size and shape based on reference
materials of scientific journals related to cultural
heritage.
(1) Start screen
(2) 360 degree drone image
(3) Experience Combine Scenario Event
(4) VR Experience Scenario

\section{Approach of VR Development}

\subsection{Reality Data Gathering}

\subsubsection{Satellite Capture Data}

The first step in the 3D restoration of lost cultural heritage is to consider the full concept of cultural heritage restoration. What is the scope of restoration? What is the actual location and exact size of each component of the cultural heritage? In order to obtain the results to this question, the topographical structure in which the cultural heritage is located should be given priority over the entire terrain. It is possible to take a close shot of a large area at once using a satellite. Unity World 
Composer is used to extract Heightmap and Image map. There are several ways to increase 3D spatial resolution. In this paper, based on satellite map, we generate basic data to refer to $3 \mathrm{D}$ terrain data.
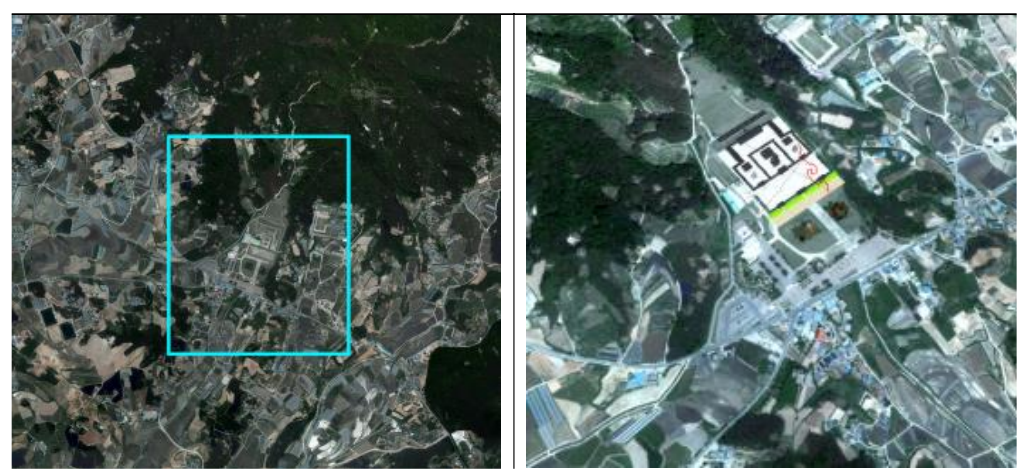

Figure 2. Unity World Composer Satellite Map

\subsubsection{Drone Shooting Data}

Based on a satellite map taken by a satellite, a scenario of a drone shooting is set up to set the restoration scope of the cultural heritage. Camera resolution is taken at $4 \mathrm{~K}$ or more, and basic work is done to create 3D terrain data, size, and location creation data.
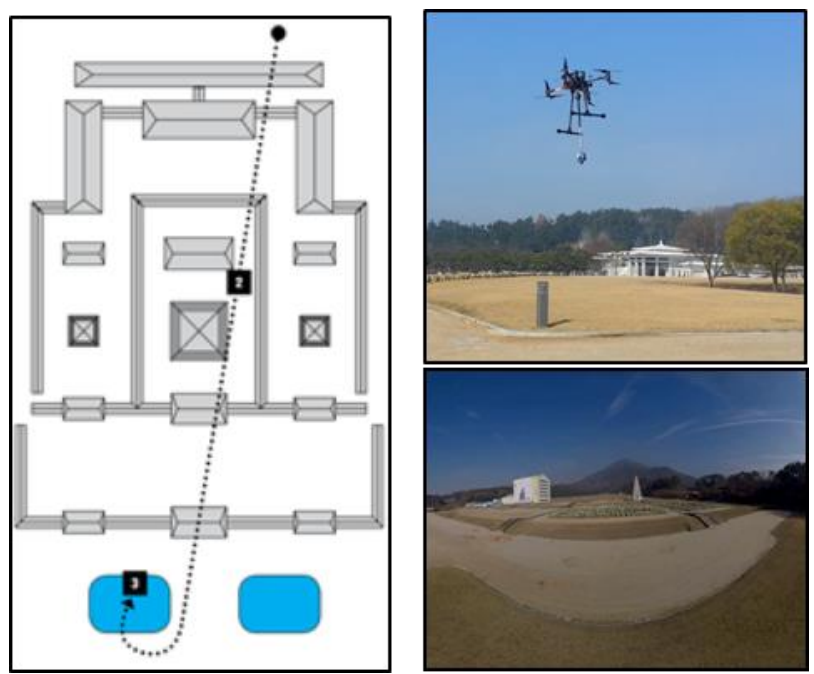

Figure 3. Drone Shooting Scenario

\subsubsection{D Map Development}

It is a fact that we should create a virtual space based on the recorded documents, reconstructed data, and historical research data in their present state. Therefore, there is a problem in how to produce the topography around the cultural relics. Among the various map production methods, the production method in this paper is extracted and applied to the terrain Heightmap data of the area using the Unity Engine. In order to use the extracted terrain Heightmap data for VR contents, it is important to grasp the shape of the entire terrain by applying it to the polygon. In this process, a large number of faces are generated. In order to reduce the number of faces, Polygon Reduction function is used to reduce the number of faces. If the faces of the parts without a height difference are made into a single face, data suitable for VR contents can be obtained. The total area of the surrounding terrain data of the 
applied cultural heritage site is set in the range of $2 \mathrm{~km}$ in length and $500 \mathrm{~m}$ in length. The shape of the area without bends was made by a Map Wire with a rectangle using at least 4 Vertices. In order to compensate for the disadvantages of the single plane of the four vertexes, the Normal Map was applied to complete the surrounding terrain.

By applying the virtual 3D architecture to the current photographed image through the inspection process, the surrounding environment and buildings close to the actual observation are produced. The extracted terrain map size is $4 \mathrm{k}$, which is not suitable for VR contents. In order to perform real-time rendering, it is necessary to reduce the capacity of the map data. A lot of effort is needed to reduce the minimal map image loss in this process. It is important to reduce the number of polygons for real-time rendering of virtual environments, but the quality of rendering depends on the resolution of the texture. In order to enhance the quality of rendering, Vertex Color is applied to express color and texture, Normal Texture is applied to express the details of object, Highlight Texture is applied to make material representing light reflection, and an environmental texture is used for expressing surrounding reflections.
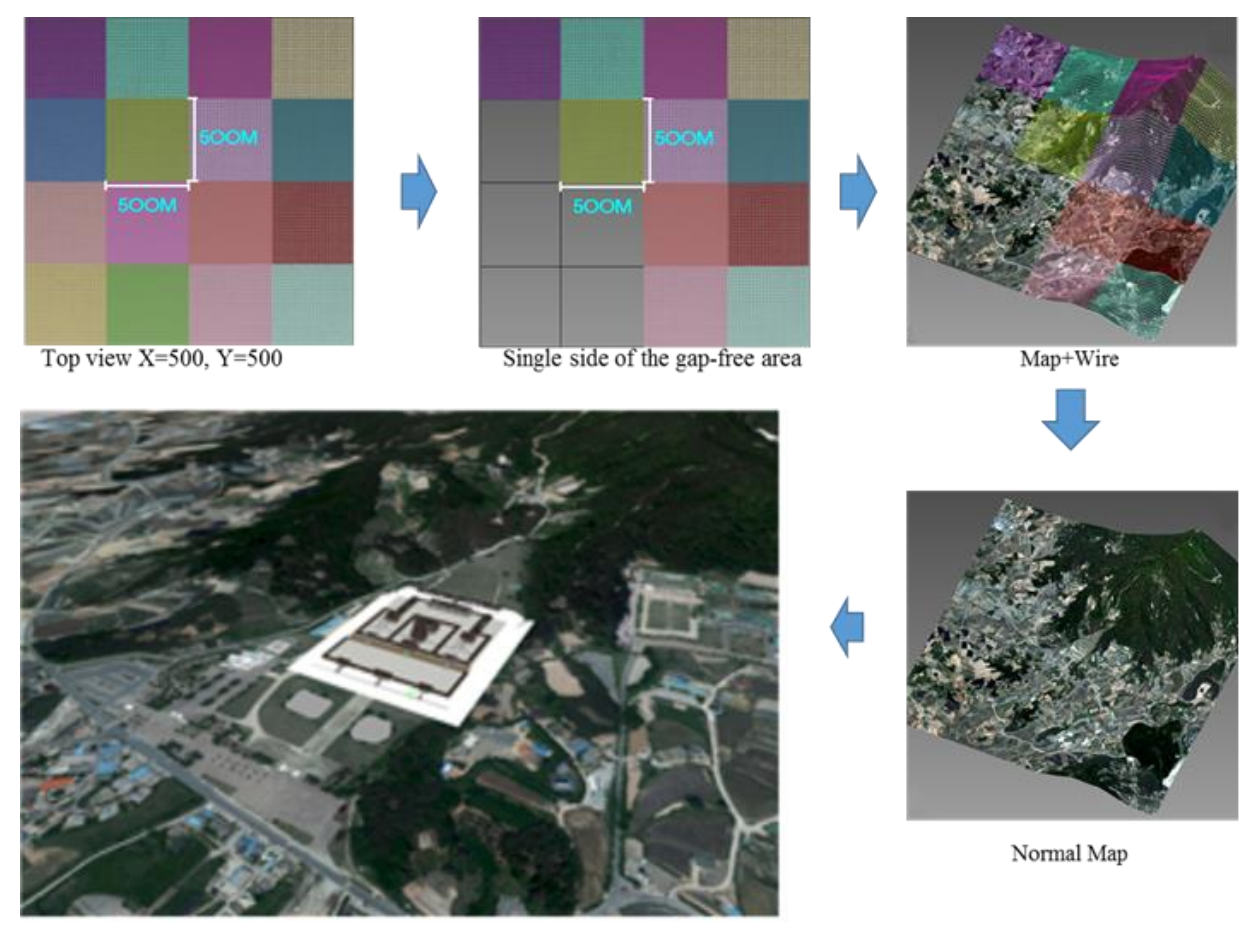

Normal Map

Virtual 3D Mapp

Figure 4. Process of 3D Map Development

Here we use the Normal Map as a way to reduce the volume of data. The smallest unit of polygons used to represent three-dimensional computer graphics is called a polygon. In 3D computer graphics, multiple vertex are connected to represent a three-dimensional object. The more polygons you use, the finer is the representation of objects. However, this 'high polygon' has the disadvantage that it takes longer to work because of the increase in the calculation time instead of the detailed representation of the object. In order to offset the disadvantage of use of such a high polygon, the Normal Map information is extracted from the high polygon and then the Normal Map information is applied to low polygon modeling. Modeling with the Normal Map shows a similar quality to that of the high polygon with a small number of polygons. 


\subsection{D Modeling}

\subsubsection{Building \& Pagoda Modeling}

Building modeling work is based on collected cultural relics model photographs, literature, and reference material. In fact, there was a lot of difficulty in modeling because of the lack of reference data. As a preparatory work for modeling the exact size and proportions, it is necessary to additionally check the size of the buildings using BOX on an actual photographed map.
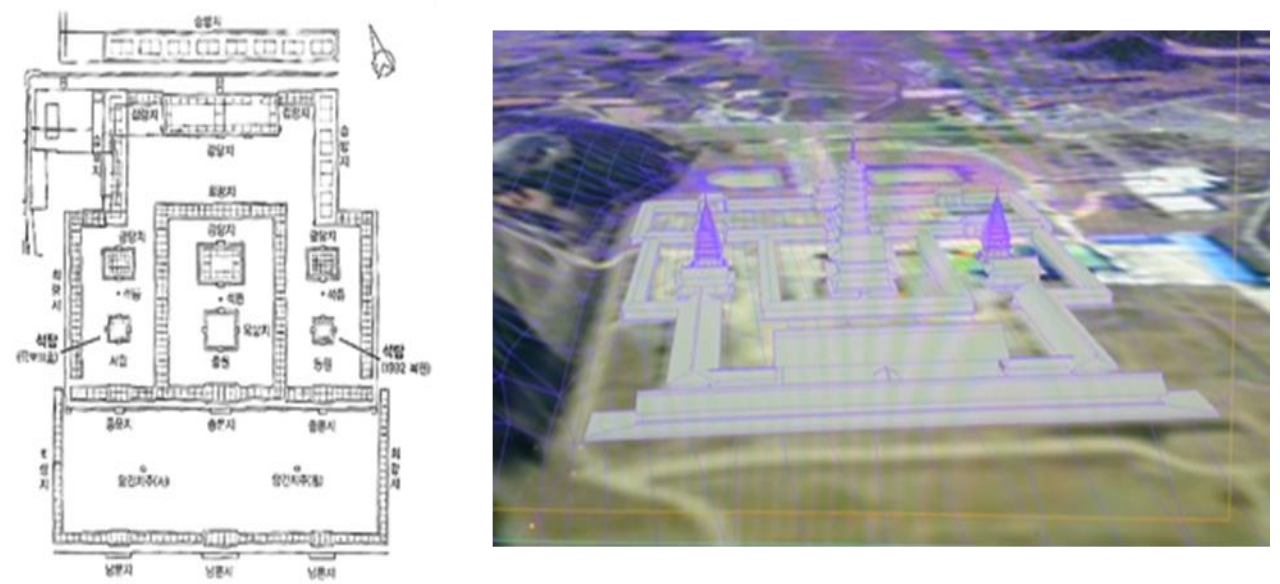

Figure 5. Cultural Heritage Data and Virtual Terrain, Building Layout Examples

Table 2. Polygons Example (Korea Miruksaji Case)

\begin{tabular}{|c|c|}
\hline Name & No. of Polygon \\
\hline Central wooden tower & 140,000 \\
\hline Stone Pagoda & 6,000 \\
\hline Center door ground (left) & 10,000 \\
\hline South door ground (center) & 32,000 \\
\hline South door ground (left) & 13,000 \\
\hline Main building of temple (center) & 14,000 \\
\hline Flagpole & 7,000 \\
\hline Vihara ,corridor ground & 46,000 \\
\hline Corridor ground (left) & 21,000 \\
\hline Corridor ground (right) & 9,000 \\
\hline Corridor ground (center) & 80,000 \\
\hline Vihara(back) & 50,000 \\
\hline
\end{tabular}

The actual working distance was set in meters, and the work was carried out. The front and back lengths were 130 meters. In order to work on 3D architecture according to the present terrain environment, this study edited the photo materials. The following is an example of the number of polygons in cultural heritage applied to VR content. Total applied cultural heritage Polygons total 428,000. 
Table 3. Modeling Develop Process

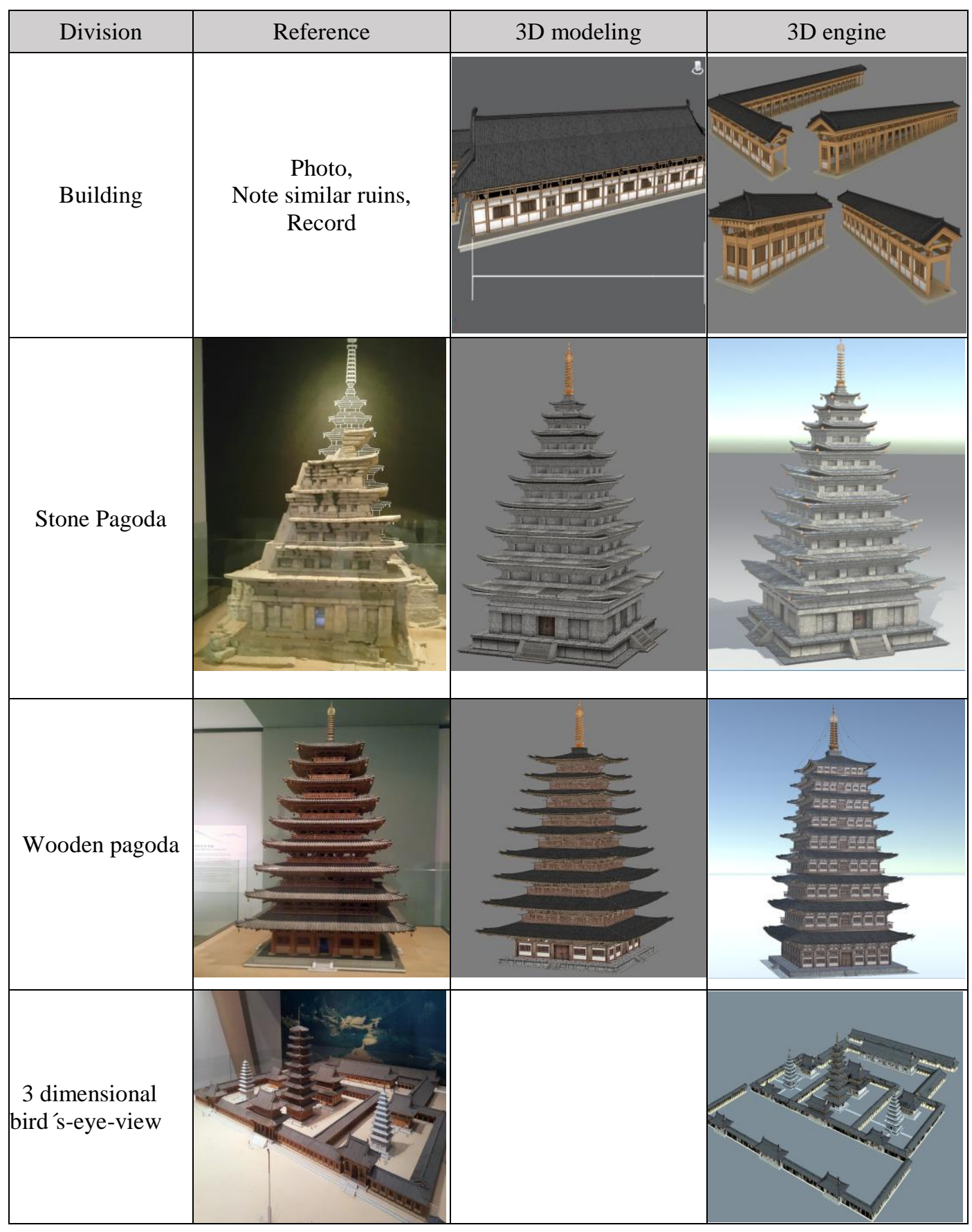

A 3D virtual building is placed on the set image, and the number of window frames on the wall of the building is measured by actual measurement. The work that requires sophistication is modeled on the basis of a lot of data because each pattern, connection part model, etc. are connected to the pillar. 3D modeling is based on model data, photographs, records, and similar references, and is implemented as virtualized content through a $3 \mathrm{D}$ engine.

\subsubsection{Mapping}

In order to respond in real time, textures should be created as a repeated tiling map to reduce resources. Instead of reducing resources with a tiling map, the size of the map is 
enlarged to increase the VR resolution. This is because the viewer experiences the closest experience in the virtual space, not the image, so the resolution of the screen has a high sensitivity to the viewer. Therefore, the size of the map should be made larger than 2048. Smaller maps may reduce resource capacity, but lower VR resolution can reduce the immersive experience.

Table 4. Applied Mapping

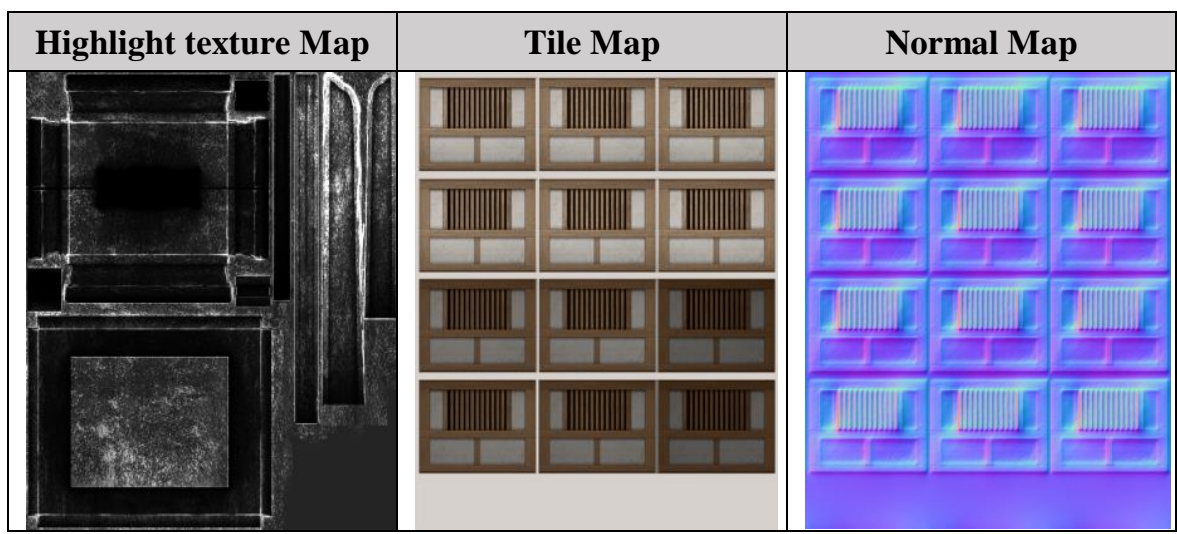

\subsubsection{Character}

\subsubsection{Character Modeling}

We make the structure of the polygon, convert it into a wire structure, and apply the map to model the character. No matter how simple a low polygon character is, the wire structure must be cleaned up to make the character's natural structure. The structure of a radial polygon should be followed based on the character's ear / eyes / mouth / nose. When creating a variety of facial expressions, it is possible to create the most natural form of muscle flow in the face. It should be made with a radial structure because there are many mouth and eye movements.
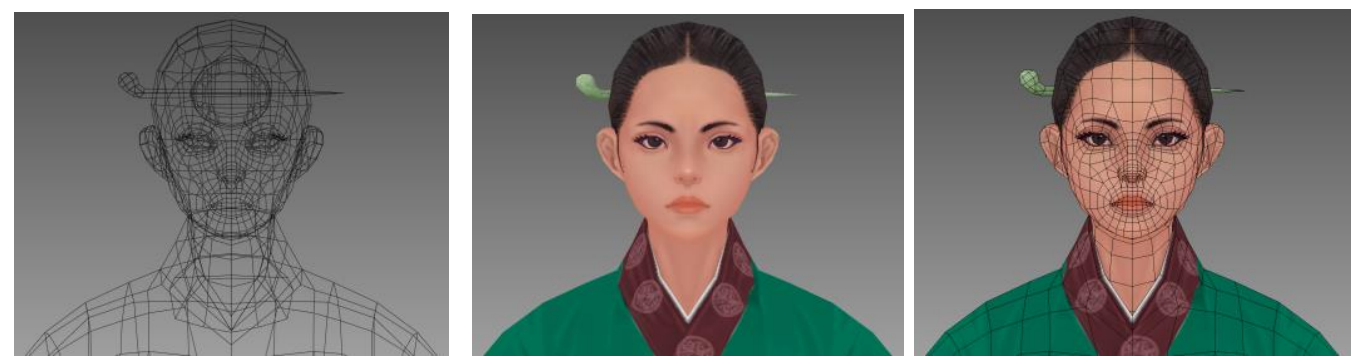

Figure 6. Process of Character Modeling

\subsubsection{Character Mapping}

\section{Step 1. Diffuse Mapping}

We use a real photo source to make the character face real. In order to make a suitable expression for each part, several photograph sources are prepared, and the texture expression of the skin and the mapping of each part are finely performed

\section{Step 2. Normal Bump Mapping}

Representative characters require a high level of modeling and proceed with Normal mapping, which allows high quality modeling with a small number of polygons. 


\section{Step 3. Ambient Occlusion Mapping}

The effectiveness of the calculation of light through global illumination is very high. A point on the surface of an object represents the amount of ambient light it accepts. The visual representation of the accepted amount of information of the light according to the position changes.

Table 5. Mapping Method Comparison

\begin{tabular}{|c|c|c|c|}
\hline $\begin{array}{l}\text { Divi } \\
\text { sion }\end{array}$ & Diffuse Map & Normal Bump Map & $\begin{array}{c}\text { Ambient Occlusion } \\
\text { Map }\end{array}$ \\
\hline \multicolumn{4}{|l|}{$\begin{array}{l}\text { Exp } \\
\text { ressi } \\
\text { on }\end{array}$} \\
\hline $\begin{array}{l}\text { Adv } \\
\text { anta } \\
\text { ges }\end{array}$ & $\begin{array}{l}\text { - Wrinkles and facial } \\
\text { expressions that match the } \\
\text { character's age using a } \\
\text { realistic map. }\end{array}$ & $\begin{array}{l}\text { - High quality rendering } \\
\text { can be achieved with a } \\
\text { small number of } \\
\text { polygons. }\end{array}$ & $\begin{array}{l}\text { - It is possible to express } \\
\text { light with the calculation } \\
\text { of light through global } \\
\text { illumination. } \\
\text { - the amount of how much } \\
\text { of the Ambient Light is } \\
\text { accepted by a point on } \\
\text { the surface of the object. }\end{array}$ \\
\hline $\begin{array}{l}\text { Disa } \\
\text { dva } \\
\text { ntag } \\
\text { es }\end{array}$ & $\begin{array}{l}\text { - Due to the fact that the } \\
\text { image of the real photo } \\
\text { source contains } \\
\text { highlights and shadows } \\
\text { defined in the image } \\
\text { itself, there is a sense of } \\
\text { heterogeneity when the } \\
\text { light is applied to the } \\
\text { engine. }\end{array}$ & $\begin{array}{l}\text { - Takes time for high } \\
\text { polygon operation to } \\
\text { create Normal Map.. } \\
\text { - Extracts Normal } \\
\text { information after working } \\
\text { with high polygons, and } \\
\text { performs additional low } \\
\text { polygon operations to } \\
\text { apply a high level of } \\
\text { extracted Normal } \\
\text { information. }\end{array}$ & $\begin{array}{l}\text { - } \text { Time required for high } \\
\text { polygon operation to } \\
\text { create AO Map is } \\
\text { required. }\end{array}$ \\
\hline 보완 & \multicolumn{3}{|c|}{$\begin{array}{l}\text { - MapSource mapped with Spotlight is created in Photoshop using Hue / Saturation to } \\
\text { create an image in black and white. High pass of filter and level of image are used to } \\
\text { make wrinkles and to make wrinkles clearly visible. } \\
\text { - Edit the MapSource and photo map extracted with Polypaint to solve the problem and } \\
\text { find the desired skin color. } \\
\text { - Various Normal map production 'Program' utilization }\end{array}$} \\
\hline
\end{tabular}

\subsubsection{Clothes \& Accessories}

Costumes are made on the basis of relevant reference books and artifacts. Instead of reducing the number of polygons as much as possible, the expression of wrinkles and delicate patterns was created based on the materials of the same period. These cases have used about 5,000 polygons in the character, and we used several techniques to make several maps using UV coordinates repeatedly. Color was used to reproduce the lifestyle of the age by expressing various clothes according to the changes in color. Especially, a large number of polygons are required for an accurate modeling of king and queen decorations. 

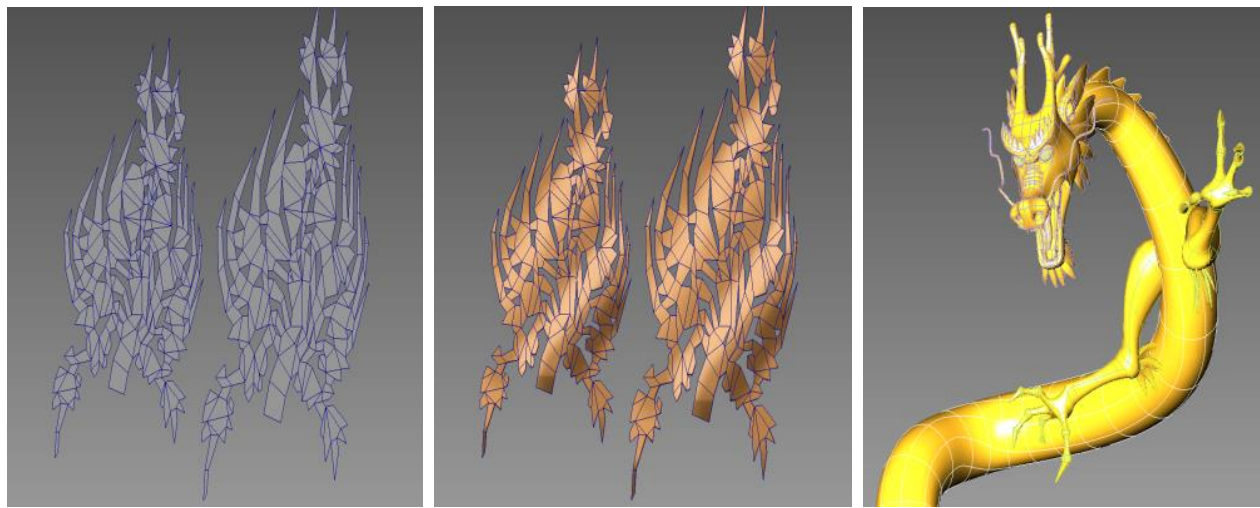

Figure 7. Accessories Modeling

\subsection{Unity Program}

Unity MapSource has applied Ambient Occlusion Map, Diffuse Map and Normal Map. Unity has applied metal highlights that make it possible to display on the same time line as real time light. In Max, vertex color is applied to AOA, and vertex color applied to vertex is applied to unity. A detailed map has been applied to simplify the development of color shades and Normal. In this case, the UV map must be unfolded in only one direction to apply the detail map.

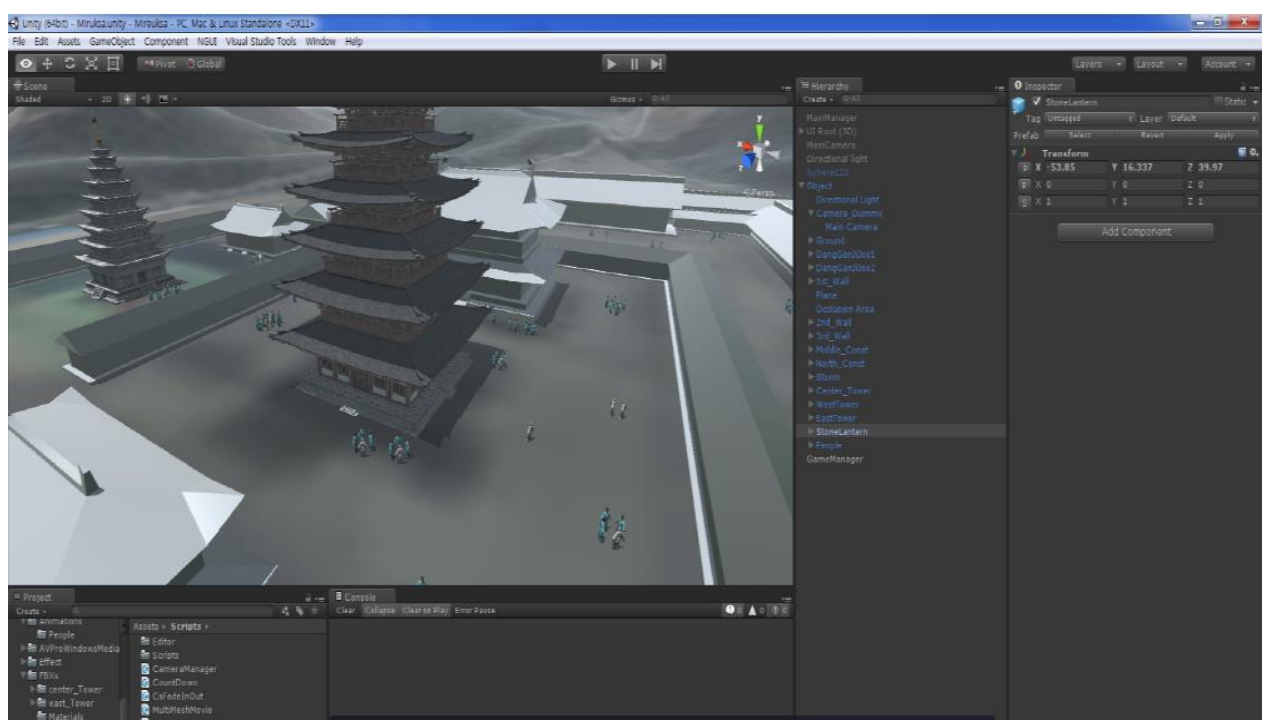

Figure 8. Unity Program

\section{VR Experience Program Code}

As a method to complement the disadvantages of restoration of existing relics, we have developed an experiential program based on interaction with the user as seen from the viewpoint of the user. The interaction technique with the user is based on interaction with VR equipment. In order to give the impression that the user walks through all the sections of the ruins, movement is applied to each event area from the entrance to the movement. At the time of development, this study used the latest version of Oculus DK2 (for developers), which caused users to feel dizzy. This problem (dizziness) was eliminated by the usage of fade in and out effects to transition to the next event area according to the progress of the event. 
Table 6. Move to Event Algorithm

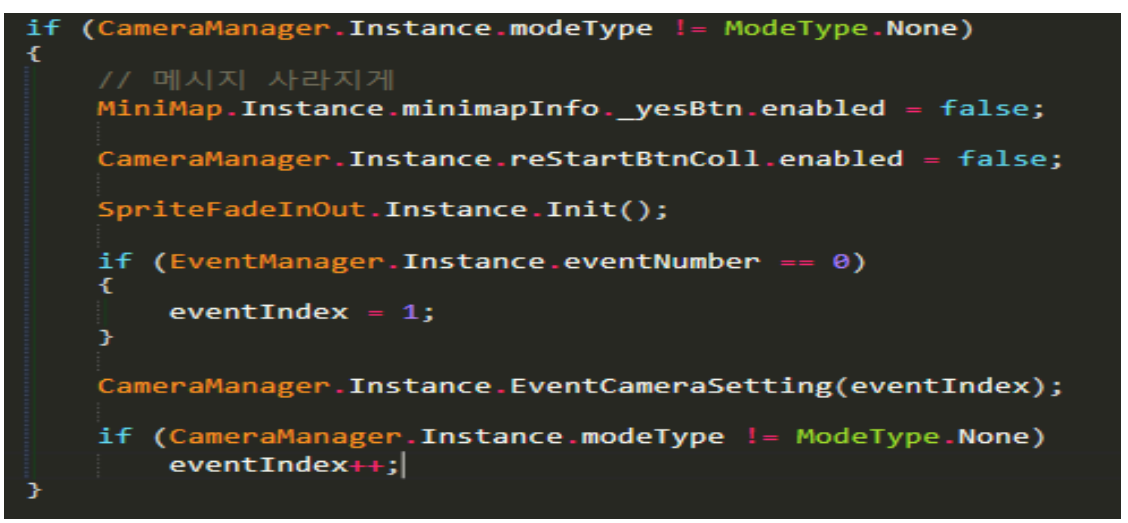

In the part where no dizziness is felt, the $3 \mathrm{D}$ object reconstructed virtually is processed so that it can be viewed in detail. In this paper, we apply restoration and VR experience to the Mireuksaji ruins in Korea as an example. It was photographed with a $360^{\circ}$ drone and cross fade with the restored Mireuksaji ruins. We interact with the user accurately and maximize the experiential element by adding an event element (interaction with the user) so that the restored $3 \mathrm{D}$ object can be seen. We added an event element so that we could see the restored 3D object while the event was going on.

\section{Table 7. Interactive Event Algorithm}

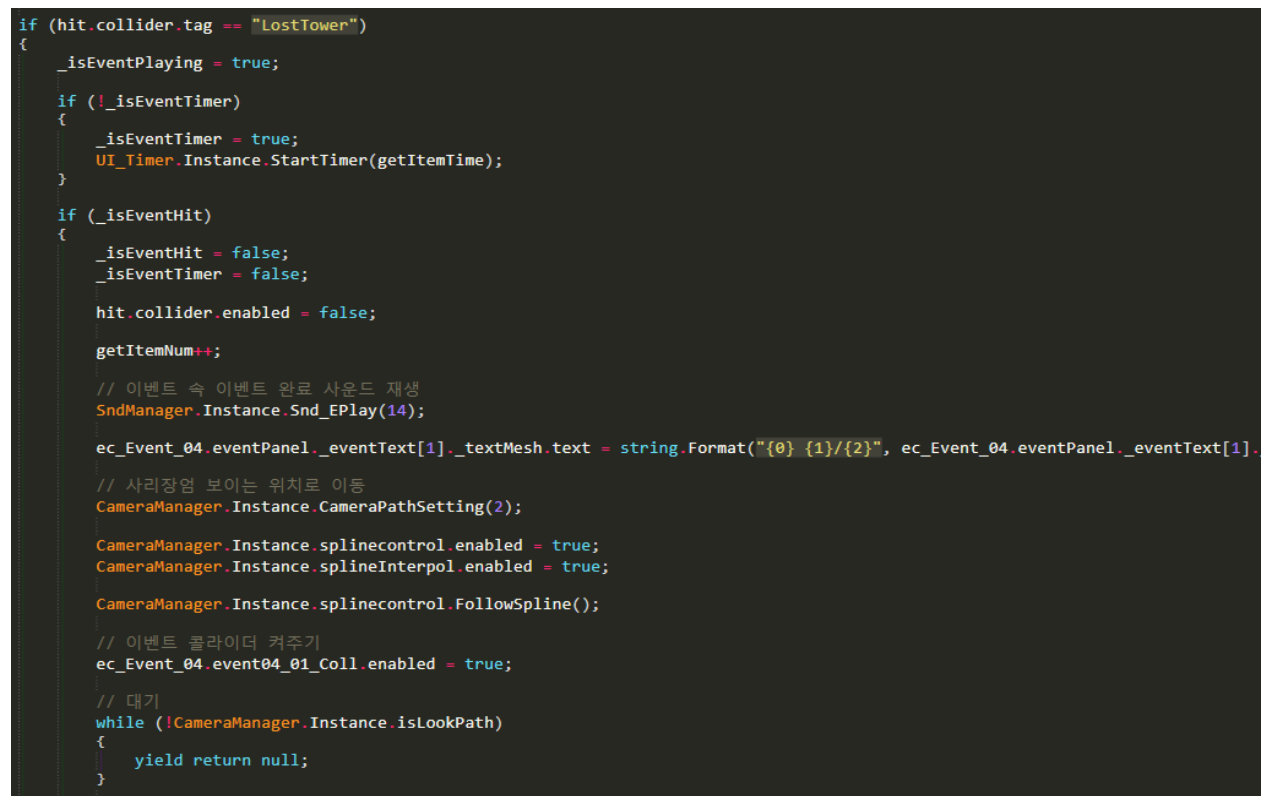

\section{Conclusion}

This paper is a study that maximizes the utilization of cultural heritage. The majority of studies are focused on restoring cultural heritage. Research that takes advantage of the cultural heritage and propagation situation is insufficient. This paper is a study that maximizes the two elements of restoration and experience of damaged cultural heritage. Applied research approaches are compared in various ways to existing methods. 
Table 8. Comparison of Existing and New Methods

\begin{tabular}{|c|c|c|}
\hline & Existing method & New way \\
\hline $\begin{array}{l}\text { Precision } \\
\text { measurement. }\end{array}$ & $\begin{array}{l}\text { Drones were photographed without } \\
\text { consideration of size, area, location, } \\
\text { etc., and were not sufficiently restored }\end{array}$ & $\begin{array}{l}\text {-We conducted satellite photography to } \\
\text { precisely measure the actual location } \\
\text { and size of the cultural heritage. } \\
\text {-Based on satellite-based data, we set } \\
\text { the scope of the remains and scenarios } \\
\text { of the drone shooting. }\end{array}$ \\
\hline \multirow[t]{5}{*}{$\begin{array}{l}\text { Modeling } \\
\text { work }\end{array}$} & 3D scanning work & $\begin{array}{l}\text { Combination of Unity, Mapping, and } \\
\text { Rendering. }\end{array}$ \\
\hline & $\begin{array}{l}\text { Number of polygons increased to } \\
\text { infinity (mesh structure complexity). }\end{array}$ & $\begin{array}{l}\text { Polygon number is } 1 / 10 \text { of the level of } \\
\text { 3D scanning operation (mesh structure } \\
\text { simplicity). }\end{array}$ \\
\hline & $\begin{array}{l}\text { Real time rendering is difficult, so only } \\
\text { static work in simple display form is } \\
\text { possible. }\end{array}$ & $\begin{array}{l}\text { Real-time rendering is possible, } \\
\text { enabling dynamic work. }\end{array}$ \\
\hline & $\begin{array}{l}\text { Interactive } \\
\text { difficult to inperience } \\
\text { experiences. }\end{array}$ & $\begin{array}{l}\text { Interactive modeling in real time makes } \\
\text { it easy to introduce game techniques. }\end{array}$ \\
\hline & & $\begin{array}{l}\text { Using a lighting function, it can be } \\
\text { expressed in real time. }\end{array}$ \\
\hline Restore & $\begin{array}{l}\text {-Restore heritage with 3D scanning data } \\
\text {-Difficult to restore damaged or lost } \\
\text { parts } \\
\text {-Requires complex data structures and } \\
\text { extensive equipment }\end{array}$ & $\begin{array}{l}\text { - Restore Using the SW based on the } \\
\text { due diligence and documentary heritage. } \\
\text { - Ability to restore 3D scanning levels. } \\
\text { - Can be applied to lost cultural heritage } \\
\text { due to events such as war, disaster (fire, } \\
\text { earthquake, damage). } \\
\text { - It is possible to experience it in } \\
\text { connection with the surrounding } \\
\text { topography of the cultural heritage and } \\
\text { the life of the time. }\end{array}$ \\
\hline
\end{tabular}

Combining the life of that era and the surrounding heritage based on empirical data, led to a comprehensive cultural heritage. Studies also introduced a solution to an imaging system that had caused dizziness arising from movement in the VR image. This needs to be verified by further studies and by surveys measuring user satisfaction completed by users of the actual application.

\section{References}

[1] J. Waleed Salish, "Imperceptible 3D Video Watermarking Technique Based on Scene Change Detection”, International Journal of Advanced Science and Technology, vol. 82, (2015), pp. 11-22.

[2] D. Throsby, "Economics and Culture", Cambridge University Press, (2001).

[3] D. Harvey, "Heritage pasts and heritage presents: temporality, meaning and the scope of heritage studies", International Journal of Heritage Studies, vol. 7, no. 4, (2001), pp. 319-338.

[4] J. H. Shun and S. J. Lee, "3D Image and Sound Control Method for User-Perceived 3D Feeling and Functional Game Optimization", International Journal of Control and Automation, vol. 7, no. 1, (2014), pp. 281-294.

[5] S. W. Ko, "Readability Improvement Method on Ancient Inscription Using Laser Scanning and Image Processing Techniques", Human Contents, vol. 21, (2011), pp. 177-198.

[6] J. O. Kim, "Real-Time Hand Gesture-Based Interaction with Objects in 3D Virtual Environments", International Journal of Multimedia and Ubiquitous Engineering, vol. 8, no. 6, (2013), pp. 339-348.

[7] J. S. Han and G. H. Lee, "VR Tourism Content Using the HMD Device", The Korea Contents Association, vol. 15, no. 3, (2015), pp. 40-47.

[8] B. Fleming, "Advanced 3D Photorealism Techniques", John Wiley \& Sons, Inc., (1999).

[9] A. Z. Aktas and E. Orcun, "A survey of computer game development, The journal of defense modeling and simulation", JDMS: a publication of the Society for Modeling and Simulation International, vol. 13, no. 2, (2016), pp. 239-251. 
[10] N. Ju hyun, "A Case Study of 3D Animation for Digital Restoration of Cultural Assets -Focused on the Silla Wolsong Reconstructed Image", The Resurrection of the Millennium" Journal of the Korean Society of Design Culture, vol. 19, no. 4, (2013) December, pp. 269-281.

[11] K. Sugihara and J. Kikata, "Automatic Generation of 3D Building Models from Complicated Building Polygons", Journal of computing in civil engineering, vol. 27, no. 5, (2013), pp. 476-488.

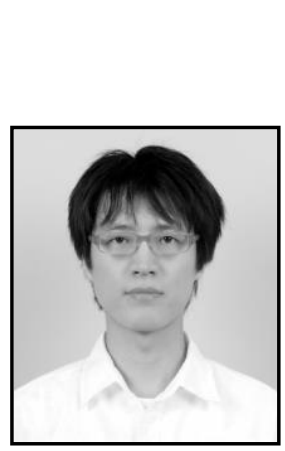

\section{Authors}

Ki-Hwan Ko is currently a professor in the department of Game Contents in the College of Culture Convergence at Jeonju University. His research interests include Computer Graphics, 3D animation, Game contents and Travel contents.

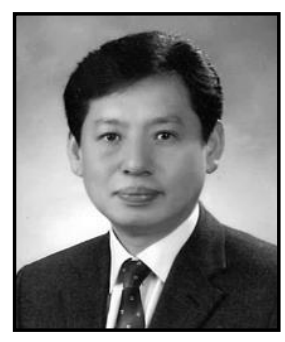

Jeong-Ik Min received MS, and Ph.D degrees in the Division of Industrial Engineering from the Korea Advanced Institute of Science and Technology (KAIST). He worked in LG CNS, IBM korea, SAP korea and Korea Telecom at 24years. He is currently a professor in the department of Smart Media in the College of Culture Convergence at Jeonju University. His research interests include IT governance, Manufacturing operating systems, Culture, Travel and Smart Farm contents. 
International Journal of $u$ - and e- Service, Science and Technology Vol. 10, No.5 (2017) 